\title{
Neonatal Herpes Encephalitis: A Case Series and Review of Clinical Presentation
}

\author{
Cory Toth, Sheri Harder, Jerome Yager
}

\begin{abstract}
Objective: To describe the clinical and laboratory findings in cases of neonatal herpes simplex virus (HSV) encephalitis. Background: Neonatal HSV encephalitis is a devastating infection which requires a high degree of clinical suspicion and rapid initiation of antiviral therapy. Methods: We performed a retrospective search for all cases of HSV encephalitis within the two Saskatchewan pediatric tertiary care centers for the period of 1985-2001. Only those patients with consistent clinical presentations along with direct evidence of presence of HSV, such as positive cerebrospinal fluid (CSF) viral cultures, positive polymerase chain reaction (PCR) for HSV from CSF, or positive immunoglobulin G against HSV from neonatal blood, were selected. Results: Five male and four female infant patients were identified. At a mean age of presentation of $24 \pm 20$ days, seizures occurred in six neonates, lethargy in six neonates, temperature changes in five neonates, and apnea in three neonates. Examination of CSF demonstrated an initial monocytosis or lymphocytosis, elevated CSF protein and depressed CSF glucose in $100 \%$ of patients. Electroencephalography (EEG) was abnormal in 100\% of patients. Initial computerized tomography was abnormal in $55 \%$ of patients. Clinical follow-up over an average of two years demonstrated developmental delay in four patients and upper motor neuron findings in four patients. No patients suffered from postencephalitic epilepsy or mortality. Conclusions: Neonatal HSV encephalitis most commonly presents with seizures, lethargy, and dysthermia. Cerebrospinal fluid testing and EEG have $100 \%$ sensitivity in cases with laboratory confirmation of HSV presence. Improvements in morbidity and mortality as compared to previous reports may relate to better recognition of this illness and acyclovir therapy. The lack of postinfection epilepsy in our series may also relate to better recognition and acyclovir therapy within this series of patients.
\end{abstract}

RÉSUMÉ: Encéphalite herpétique néonatale: étude de cas et revue du tableau clinique. Objectif: Décrire les observations cliniques et biochimiques chez les patients atteints d'encéphalite néonatale causée par le virus de l'herpès simplex (VHS). Contexte: Dans l'encéphalite néonatale à VHS, une infection dévastatrice, on doit faire preuve d'une grande acuité diagnostique et instituer promptement un traitement antiviral. Méthodes: Nous avons fait une recherche rétrospective de tous les cas d'encéphalite à VHS dans les deux centres pédiatriques de soins tertiaire de la Saskatchewan de 1985 à 2001. Seuls les patients dont le tableau clinique était conforme à celui de l'encéphalite à VHS et chez qui la présence du VHS avait été démontrée soit par une culture positive du liquide céphalo-rachidien (LCR), une amplification en chaîne par polymérase positive du LCR ou des immunoglobulines G anti-VHS positives dans le sang ont été sélectionnés. Résultats: Neuf bébés, soit cinq garçons et quatre filles, ont été identifiés. L’âge moyen de présentation était de $24 \pm 20$ jours et les symptômes initiaux ont été des convulsions chez six, de la léthargie chez six, des variations de température chez cinq et de l'apnée chez trois. L'examen du LCR a montré initialement une monocytose ou une lymphocytose, une élévation des protéines et une baisse du glucose chez tous les patients. L'électroencéphalogramme était anormal chez tous. La tomodensitométrie initiale était anormale chez 55\% des patients. Le suivi clinique sur une période moyenne de deux ans a montré un retard de développement chez quatre patients et des anomalies du neurone moteur supérieur chez quatre patients. Aucun patient ne présentait de l'épilepsie postencéphalitique et il n'y a eu aucun décès. Conclusions: L'encéphalite néonatale à VHS se manifeste la plupart du temps par des convulsions, de la léthargie et de la dysthermie. L'examen du LCR et l'ÉEG ont une sensibilité de 100\% chez les cas où il y a confirmation de la présence du VHS par le laboratoire. L'amélioration de la morbidité et de la mortalité par rapport aux observations antérieures est sans doute due à une meilleure identification de la maladie et au traitement par l'acyclovir. L'absence d'épilepsie postinfectieuse dans notre série de cas peut également être due à une meilleure identification de la maladie et au traitement par l'acyclovir chez ces patients.

Can. J. Neurol. Sci. 2003; 36-40

Neonatal herpes simplex virus encephalitis (HSV-E) was first described in the $1930 \mathrm{~s},{ }^{1}$ centuries after descriptions of the adult form of HSV-E were available. Three decades later, viral cultures and immunologic techniques permitted herpes simplex virus-1 (HSV-1) to be distinguished from herpes simplex virus-2 (HSV$2){ }^{2}$ These techniques allowed for clearer definitions of epidemiology and pathogenesis of HSV-1 and HSV-2 infections. ${ }^{3}$ The prognosis for the two viral types varies as well, with HSV-1 infection associated with a better overall prognosis than with HSV-2 as a cause of neonatal encephalitis. ${ }^{4}$

Because HSV-2 resides in the genital tract, neonatal herpes simplex virus (HSV) infections are most commonly acquired via ascending intrauterine infection, exposure to an infected maternal birth canal, or postnatal infection. ${ }^{5}$ It has been estimated that $75-86 \%$ of neonatal HSV-E cases are due to HSV-2. ${ }^{4}$ In contrast, childhood

From the Division of Neurology, Department of Medicine (CT), Department of Radiology (SH), and Division of Pediatric Neurology, Department of Pediatrics (JY), University of Saskatchewan, Saskatoon, Saskatchewan, Canada.

Received February 5, 2001. AcCePted in final fORm August 12, 2002. Reprint requests to: Cory Toth, University of Calgary, Department of Clinical Neurosciences, 3330 Hospital Drive NW, Calgary, Alberta, Canada T2N 4N1 
and adult HSV-E is due to HSV-1 in $99 \%$ of patients. ${ }^{6}$ While cases of neonatal HSV-E due to HSV-1 are uncommon, they may occur via vertical transmission during a primary maternal infection of HSV-1. In cases of neonatal HSV-E due to HSV-2 exposure during delivery, a latency of typically 11-17 days following delivery has been described prior to clinical presentation. ${ }^{5}$

Neonatal HSV-E can present with multiple clinical features, including fever or temperature fluctuations, tense fontanelle, seizures and coma. ${ }^{5}$ In the absence of antiviral and supportive therapy, mortality has been previously estimated to be between $40-50 \%$. Neonatal survivors have been described in the literature to have high rates of morbidity, including mental retardation, seizures, impaired growth, retinopathy, and cystic encephalomalacia. $^{5}$

We have examined our experience with neonatal HSV-E in Saskatchewan over the past 16 years, in order to determine the clinical presentation, sensitivity of diagnostic procedures, efficacy of antiviral therapeutics, and prognosis in all patients with compatible clinical features and positive laboratory evidence of HSV.

\section{MATERIALS AND METHODS}

We performed a retrospective search from hospital records at the Royal University Hospital, Saskatoon, Saskatchewan, and the Regina General Hospital, Regina, Saskatchewan, for all neonatal patients diagnosed as "encephalitis" or "herpes encephalitis" within the period of 1985-2001. Saskatchewan has a population of approximately one million, and averaged 12,000 live births per year over the period of time from 1985-2001 (Saskatchewan Bureau of Statistics). These two hospitals are the only tertiary pediatric care centers for the province. A neonate was defined as an infant less than 28 days of age.

Inclusion criteria were composed of:

1) neonates with central nervous system dysfunction consistent with a clinical diagnosis of encephalitis; and

2) laboratory confirmation of HSV infection, including positive cerebrospinal fluid (CSF) HSV culture, positive polymerase chain reaction (PCR) for $\mathrm{HSV}$ from $\mathrm{CSF}$, or positive immunoglobulin $\mathrm{G}$ ( $\mathrm{IgG}$ ) against $\mathrm{HSV}$ from blood from the neonate.

All patients without laboratory evidence were excluded, due to the possibility that other CNS infections such as neonatal enteroviral encephalitis, bacterial sepsis and meningitis, and rare cases of congenital or neonatal cytomegalovirus and rubella infections could be causative.

The epidemiological data, premorbid status, maternal history, age of presentation, clinical presentation and course, relevant laboratory examinations consisting of CSF, blood tests, electroencephalography (EEG), computerized tomography (CT) of the brain, magnetic resonance imaging (MRI) of the brain, presence and type of therapy, and follow-up status including morbidity and mortality were recorded for each patient. Sensitivity of each investigation was calculated, with the numerator determined by presence of abnormal tests for each individual patient, and the denominator determined by the total number of patients identified.
Table 1: Clinical presentation of neonatal HSV - signs

\begin{tabular}{lc}
\hline Exam Finding & $\begin{array}{c}\text { Occurrence } \\
\text { (\% of episodes) }\end{array}$ \\
Seizure & $67 \%$ \\
Lethargy/Obtundation & $67 \%$ \\
Fever/Hypothermia & $56 \%$ \\
Poor Feeding & $44 \%$ \\
Apnea & $33 \%$ \\
Irritability & $22 \%$ \\
Respiratory Distress & $11 \%$ \\
Bulging Fontanelle & $11 \%$ \\
Asymmetrical Tone & $11 \%$ \\
Forehead Vesicles & $11 \%$ \\
Focal Clonic Movements & $11 \%$
\end{tabular}

\section{RESULTS}

A total of nine neonates, five males $(55 \%)$, were given a final diagnosis of neonatal HSV encephalitis and had laboratory confirmation of HSV within the defined period for this investigation. The mean age for these patients at time of clinical presentation was $24 \pm 20$ days. Only one neonate (11\%) was born prematurely. Only one neonate $(11 \%)$ had a maternal history of prior genital HSV identified prior to delivery. Prior asymptomatic or unidentified maternal genital HSV infections may have occurred.

Clinical presentation of affected neonates was variable, with seizure, lethargy or drowsiness, and fever or hypothermia the most common presenting symptoms or features. Other less common symptoms included poor feeding, apnea, irritability, respiratory distress, bulging of the anterior fontanelle, asymmetry of appendicular tone, forehead vesicles, and focal clonic limb movements (Table 1).

Cerebrospinal fluid examination was performed in all patients

Table 2: Cerebrospinal fluid findings in neonatal herpes encephalitis

\begin{tabular}{lcc}
\hline CSF Exam Finding & $\begin{array}{c}\text { Occurrence } \\
\text { (\% of episodes) }\end{array}$ & Mean Value \\
CSF leukocytosis & $100 \%$ & $62 \pm 64 \mathrm{cells} / \mathrm{mm}^{3}$ \\
$\quad$ CSF monocytosis & $56 \%$ & \\
$\quad$ CSF lymphocytosis & $44 \%$ & $402 \pm 650 \mathrm{cells} / \mathrm{mm}^{3}$ \\
CSF erythrocytosis & $56 \%$ & $1.5 \pm 0.6 \mathrm{~g} / \mathrm{L}$ \\
CSF protein elevation & $100 \%$ & $2.1 \pm 0.8 \mathrm{mmol} / \mathrm{L}$ \\
CSF glucose depression & $100 \%$ & \\
CSF HSV Culture Positivity & $50 \%(2$ of 4$)$ & \\
Initial CSF HSV PCR Positivity & $71 \%(5$ of 7$)$ & \\
Second CSF HSV PCR Positivity & $50 \%(1$ of 2$)$ & \\
$\begin{array}{l}\text { Immunoglobin G against HSV } \\
\text { from blood }\end{array}$ & $100 \%(2$ of 2$)$ & \\
\end{tabular}


(Table 2). Cerebrospinal fluid leukocytosis was found in $100 \%$ of patients, with both predominant monocytosis and lymphocytosis reported; CSF protein elevation and CSF glucose depression occurred in all patients; CSF erythrocytosis was reported in $56 \%$ of patients. Cultures for HSV from CSF was performed in four patients and were positive in two patients for HSV-2 (50\%). Polymerase chain reaction testing using CSF for identification of HSV was positive in five of seven patients $(71 \%)$ on the first or only PCR test performed. In cases where a second PCR for HSV within CSF was performed, positivity was $50 \%$ (one of two patients). The second PCR was performed in one patient where first PCR was positive (second PCR positive) and in one patient where first PCR was negative (second PCR negative). The laboratory where PCRs were performed was not capable of distinguishing between HSV-1 and HSV-2 using PCR. Two additional patients had diagnosis confirmed with a positive immunoglobulin $\mathrm{G}$ (IgG) against HSV found in blood from the infants.

Other investigations utilized for diagnosis of neonatal HSV-E included EEG, CT of brain, and MRI of brain. Electroencephalograhy was performed in all patients and demonstrated abnormalities in $100 \%$, with focal epileptiform discharges $(50 \%)$, burst suppression $(25 \%)$, focal electrographic seizures $(25 \%)$, focal suppression $(25 \%)$, and diffuse slowing $(25 \%)$ observed.

A CT of the brain was performed in all patients at an average of 2.3 days following clinical presentation and was repeated in five patients at an average of 9.0 days following initial clinical presentation. The CT of the brain was abnormal in $67 \%$ of patients during the period of illness, with parenchymal attenuation abnormalities (67\%), parenchymal atrophy (33\%), parenchymal contrast enhancement (33\%), leptomeningeal contrast enhancement (22\%), extra-axial fluid collection (22\%), and parenchymal calcification $(22 \%)$. Strict unilateral involvement was present in only two patients with abnormal imaging (33\%). An MRI of the brain was performed in two patients, with both patients reported with abnormal imaging (Figure 1). An MRI was performed later in the clinical course than CT scanning. In patients with both neuroimaging techniques performed (two patients), MRI images were not more sensitive than CT images in these two patients, with areas of hyperintensity and hemorrhage identified on MRI images seen on CT images as contrast enhanced regions with hypodensity and hemorrhage respectively. Figures demonstrating different neuroimaging patterns are presented in Figures 2, 3, and 4.

All patients were treated with intravenous acyclovir, and seven of nine patients $(78 \%)$ were treated with antibiotics for a minimum of two days after initial presentation before discontinuation within the subsequent four days. Five patients received phenobarbitol, one patient received dilantin, and one patient was treated prophylactically with lorazepam for control of seizures at initial presentation.

All patients have had an average follow-up of $25 \pm 20$ months with clinical assessment in all patients. Assessment included history and physical examination by a Pediatric Neurologist. Inquiry into existence of postencephalitic epilepsy was made. Audiometry was performed in $56 \%$ of patients, and showed no abnormalities. Head circumference to determine microcephaly was assessed using Center for Disease Control Growth Charts.
Table 3: Clinical follow-up of cases of neonatal HSV encephalitis (average of $25 \pm 20$ months)

\begin{tabular}{lc}
\hline Morbidity & $\begin{array}{c}\text { Occurrence } \\
\text { (\% of episodes) }\end{array}$ \\
Normal & $44 \%$ \\
Developmental Delay & $44 \%$ \\
Hemiparesis, Mild & $22 \%$ \\
Spastic Quadriplegia & $22 \%$ \\
Microcephaly & $11 \%$ \\
\hline
\end{tabular}

Overall development was assessed for gross and fine motor function, language function, cognitive ability, and social skills relative to their age, with a diagnosis of developmental delay made according to the American Academy of Pediatric Guidelines. ${ }^{7}$ Morbidity was defined as any clinical limitation of neurological abilities secondary to prior infection with herpes encephalitis. Morbidity following neonatal HSV encephalitis was identified in $56 \%$ of patients (Table 3). No cases of postencephalitis epilepsy occurred at long-term follow-up; one patient had two seizures after hospital discharge but no seizures after six months of follow-up, which has now extended to 36 months. There were no patients suffering mortality.

\section{REPRESENTATIVE CASE STUDY}

A 22-day-old premature male neonate presented with poor feeding, lethargy, brief spells of apnea, and hypothermia over two days prior to admission. There was no maternal history of herpes infection. Physical examination at admission showed obtundation with no eye opening to verbal or physical stimuli. Fontanelles were open and soft. Head circumference was 50th percentile for gestational age. Cranial nerves, tone, and reflexes were all unremarkable. All extremities were moving equally. Fundoscopy and otoscopy were normal. No meningismus was detected. Tachypnea was present with a respiratory rate of $60 /$ minute.

Endotracheal intubation was performed. Lumbar puncture was performed and revealed a monocytic pleocytosis with depressed CSF glucose and elevated CSF protein. Electroencephalography was markedly abnormal with demonstration of a burst suppression pattern.

Based upon the clinical and CSF picture, a differential diagnosis of sepsis, early bacterial meningitis, or HSV-E was considered. Acyclovir, cefotaxime, and ampicillin were started parenterally. Supportive measures consisting of airway support, ventilation, and nasogastric feeding were provided.

Polymerase chain reaction for HSV within CSF returned positive on two separate occasions. Initial CT of the brain performed on the day of admission showed only subtle subependymal contrast enhancement of the superior portions of the lateral ventricles. Follow-up CT scan of the brain fourteen days after initial presentation demonstrated left temporal lobe hypodensity with associated gyriform hyperdensity and enhancement involving the left temporal lobe, insula, and cingulate gyrus (Figure 4). Foci of acute hemorrhage were noted in the right thalamus and lentiform nucleus, right parietal lobe and left frontal lobe. A wedgeshaped region of hypodensity in the right posterior middle cerebral artery distribution was consistent with a probable vasculitic infarct. 
A
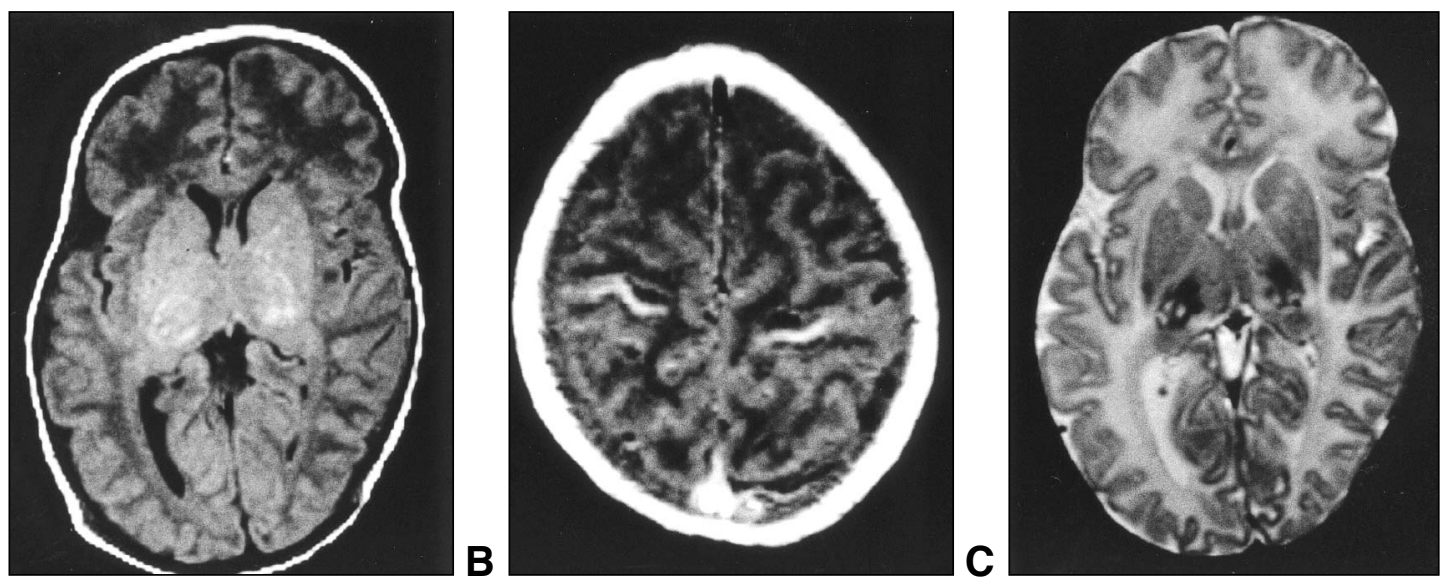

Figure 1: Unenhanced T1-weighted MRI demonstrating increased T1 signal in the thalami bilaterally (A). Gyriform hyperintensity in the high parietal lobes bilaterally $(B)$. T2-weighted MRI displaying decreased signal in the thalami bilaterally. Findings are consistent with subacute hemorrhage $(C)$.

A

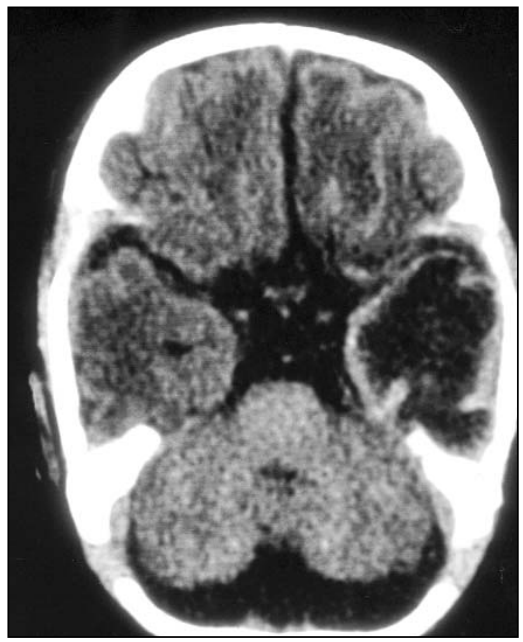

B

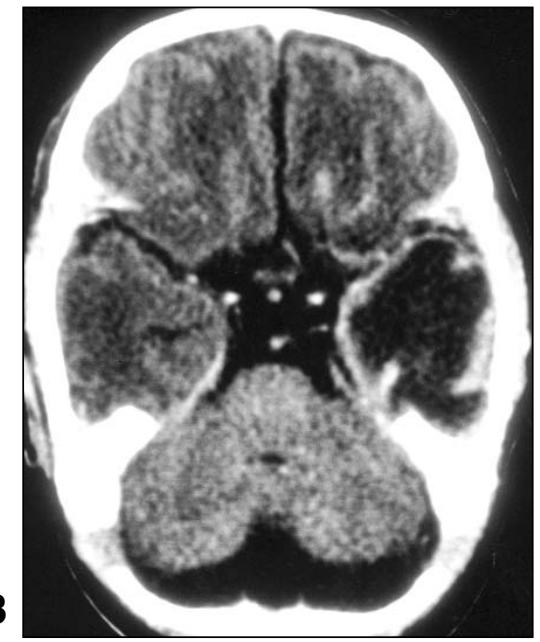

Figure 2: Unenhanced $(A)$ and enhanced $(B) C T$ demonstrating increased gyral attenuation involving the temporal lobe and inferior frontal lobe on the left with very mild enhancement after contrast administration (B).

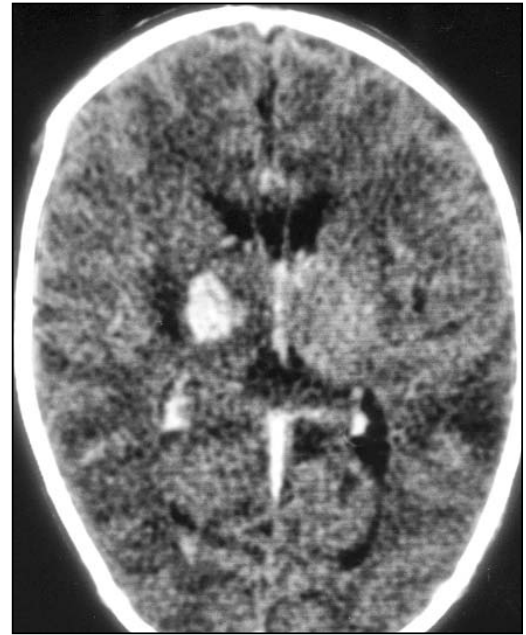

Figure 3: Unenhanced CT demonstrating hemorrhage within the right thalamus.
A



B

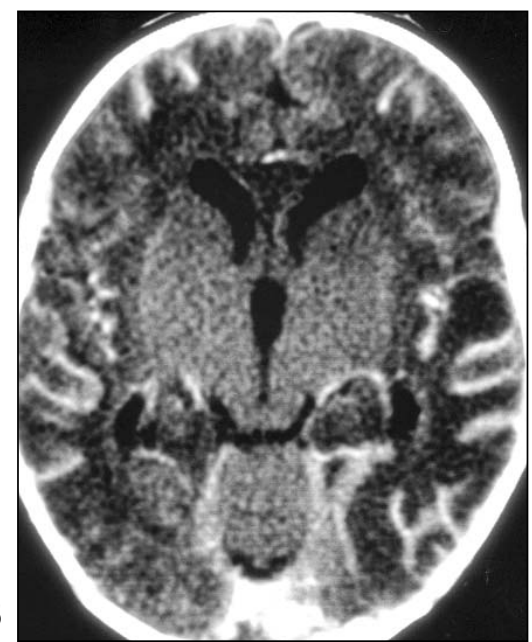

Figure 4: CT images demonstrating diffuse increased gyral attenuation on the unenhanced study (A) and diffuse gyriform enhancement after contrast administration (B). 
Clinical follow-up over six months has not demonstrated any evidence of morbidity for this patient.

\section{DiscuSsion}

Neonatal HSV-E requires a low threshold of sensitivity for diagnosis and treatment in order to best prevent early and late complications. Diagnosis of HSV encephalitis in the neonate depends upon CSF abnormalities of mild-moderately elevated white cells and red cells in CSF, depressed CSF glucose, mildmoderately elevated CSF protein and occasional CSF viral culture positivity. ${ }^{5}$ Polymerase chain reaction for HSV is highly sensitive and specific for HSV-E when compared to brain biopsy. ${ }^{8}$ In neonatal HSV-E, PCR using CSF has 67-90\% sensitivity. ${ }^{9}$ In our series, CSF examination was abnormal in $100 \%$ of patients, with PCR positive in $71 \%$ of initial tests, and $50 \%$ of repeated tests.

Past studies of HSV-E have reported EEGs to be almost uniformly abnormal, ${ }^{10}$ and in our study, sensitivity of EEG in this study was $100 \%$.

Neuroimaging results in HSV-E have been reported to differ depending upon age of the patient. Neonatal infections have been described to involve the periventricular white matter with sparing the medial temporal and frontal lobes, ${ }^{11}$ along with patchy areas of necrosis, hemorrhage and edema in a multifocal pattern. ${ }^{12,13}$ It has been reported that herpes encephalitis in neonates rarely shows hemorrhage or medial temporal or inferior frontal lobe involvement. ${ }^{14}$ However, three of six (50\%) patients in our series with neuroimaging abnormalities had evidence of hemorrhage within the brain. Prior studies of CT in neonatal HSV-E identified abnormal imaging in $56 \% .{ }^{13}$ In our series, CT of the brain performed between one and 16 days after presentation showed abnormalities with sensitivity of $67 \%$, with abnormalities in a multifocal or diffuse pattern affecting the temporal, frontal, parietal and subcortical regions.

Viral exposure during delivery has been reported to lead to a delay in clinical presentation of one to three weeks. ${ }^{5}$ However, in this series, the average latency was $24 \pm 20$ days. The difference in our reported value may be due to better laboratory recognition of HSV encephalitis, or improper reporting of the latent period previously. Prematurity has also been reported as a risk factor in the past, with $40-50 \%$ of reported neonatal HSV-E occurring in premature neonates. ${ }^{5}$ However, in this series, only one patient $(11 \%)$ was premature. This lower rate of prematurity may be due to improved fetal and maternal care over the two decades since the last report of morbidity rates in neonatal HSV-E. ${ }^{5}$ Mortality rate without specific antiviral and supportive therapy has been reported as approximately $50 \%$ in past studies. In this study, all patients received acyclovir therapy, which may explain the relative lack of mortality as compared to previous studies. Previous studies have suggested morbidity levels as high as $90 \%$, with survivors suffering from multiple sequelae including epilepsy, mental retardation, retinopathy and cystic encephalomalacia. ${ }^{5}$ In our study, $56 \%$ of patients had long-term morbidity. Only one patient $(11 \%)$ suffered from seizures six months after diagnosis, but had anticonvulsant therapy discontinued and did not suffer from epilepsy at long-term follow-up of 36 months. Improved morbidity may also have resulted from acyclovir therapy and improved neonatal care in hospital since prior reports.

\section{Conclusion}

Neonatal HSV-E requires a low threshold for clinical diagnosis and rapid initiation of antiviral therapy. Features most supportive of a clinical diagnosis of neonatal HSV-E include seizures, lethargy and dysthermia. In our series, CSF findings and EEG are $100 \%$ sensitive in patients with laboratory documentation of HSV presence. The previously reported latency of presentation post-delivery may extend beyond prior reports of one to three weeks. Long-term morbidity approximates $50 \%$ in our series, with no mortality and no cases of post-illness seizures, in contrast to prior reports. In cases of suspected neonatal HSV-E, we emphasize the need for a low threshold for clinical consideration, substantiation of the diagnosis with investigations including CSF, EEG, and cerebral imaging, followed by early treatment.

\section{ACKNOWLEDGEMENTS}

There was no financial support received for the work in this manuscript.

\section{REFERENCES}

1. Hass M. Hepatoadrenal necrosis with intranuclear inclusion bodies: report of a case. Am J Pathol 1935; 11:127.

2. Nahmias AJ, Dowdle W. Antigenic and biologic differences in herpesvirus hominis. Prog Med Virol 1968;10:110-159.

3. Nahmias AJ, Roizman B. Infection with herpes-simplex viruses 1 and 2 (parts 1, 2, and 3). N Engl J Med 1973;289:667-674,719724,781-789.

4. Corey L, Whitley RJ, Stone EF, Mohan K. Difference between herpes simplex virus type 1 and type 2 neonatal encephalitis in neurological outcome. Lancet 1988;1(8575-6):1-4.

5. Whitley RJ, Nahmias AJ, Visintine AM, Fleming CL, Alford CA. The natural history of herpes simplex virus infection of mother and newborn. Pediatrics 1980; 66:489-494.

6. Dennett C, Cleator GM, Klapper PE. HSV-1 and HSV-2 in herpes simplex encephalitis: a study of sixty-four cases in the United Kingdom. J Med Virol 1997; 53:1-3.

7. American Academy of Pediatrics Committee on Children With Disabilities. Developmental Surveillance and Screening of Infants and Young Children (RE0062). Pediatrics 2001;108:192196.

8. Lakeman FD, Whitley RJ. Diagnosis of herpes simplex encephalitis: application of polymerase chain reaction to cerebrospinal fluid from brain biopsied patients and correlation with disease. National Institute of Allergy and Infectious Diseases Collaborative Antiviral Study Group. J Infect Dis 1995; 171:857863.

9. Malm G, Forsgren M. Neonatal herpes simplex virus infections: HSV DNA in cerebrospinal fluid and serum. Arch Dis Child Fetal Neonatal Ed 1999;81:F24-F29.

10. Mizrahi EM, Tharp BR. A characteristic EEG pattern in neonatal herpes simplex encephalitis. Neurology 1982;32:1215-1220.

11. Tien RD, Felsberg GJ, Osumi AK. Herpes virus infections of the CNS: MR findings. AJR Am J Roentgenol 1993;161:167-176.

12. Davis JM, Davis RD, Kleinman GM, Kirchner HS, Taveras JM. Computerized tomography of herpes simplex encephalitis, with clinicopathological correlation. Radiology 1978;129:409-417.

13. Herman TE, Cleveland RH, Kushner DC, Taveras JM. CT of neonatal herpes encephalitis. Am J Neuroradiol 1985;6:773-775.

14. Buff B, Matthews V, Elster A. Bacterial and parenchymal infections of the brain. Top Magn Reson Imaging 1994;6:11-21. 